\title{
Temperature Dependence of High Frequency Noise Behaviors for RF MOSFETs
}

\author{
Sheng-Chun Wang, Pin Su, Member, IEEE, Kun-Ming Chen, Chien-Ting Lin, Victor Liang, and \\ Guo-Wei Huang, Member, IEEE
}

\begin{abstract}
For the first time, the temperature dependences of radio frequency $(\mathrm{RF})$ metal oxide semiconductor field effect transistors' intrinsic noise currents, including the induced gate noise current $\left(i_{g}\right)$, channel noise current $\left(i_{d}\right)$ and their correlation noise current, are experimentally investigated. The power spectral densities for the induced gate noise current and correlation noise current are found to rise as temperature increases, and decline for the channel noise current. Moreover, by using van der Ziel's noise model, our experimental results show that, besides ambient temperature, the channel conductance is the main factor dominating the RF noise behaviors. Finally, bias dependence results are also presented.
\end{abstract}

Index Terms-Metal oxide semiconductor field effect transistors (MOSFETs), noise, radio frequency (RF), temperature, van der Ziel's model.

\section{INTRODUCTION}

$\mathbf{T}$ HE noise performance of radio frequency metal oxide semiconductor field effect transistors (RF MOSFETs) is critical to RF applications, especially to the design of low noise amplifiers, resulting in a need for the accurate noise modeling [1]. Besides, it is well known that both the small-signal circuit parameters and noise sources play important roles in RF noise modeling. The temperature dependence of small-signal performance has been widely discussed [2], [3], but that of RF noise sources was deficient. Therefore, for the purpose of temperature modeling and understanding the underlying physics, the temperature dependence of noise sources demands investigation.

Pascht $e t a l$. have presented the temperature noise model by exploiting the circuit simulator [4]. However, only the channel noise source has been included, and its temperature dependence was not clear. In this letter, we will experimentally study the temperature dependence of the power spectral densities (PSDs) for the induced gate noise current $\left(S_{i g}\right)$, channel noise current $\left(S_{i d}\right)$ and their correlation noise current $\left(S_{i g d^{*}}\right)$ for the

Manuscript received February 11, 2008; revised April 11, 2008. Published August 6, 2008 (projected). This work was supported by the United Microelectronics Corporation and the National Science Council of Taiwan, R.O.C.

S.-C. Wang is with the Department of Electronics Engineering, National Chiao Tung University, Hsinchu 300, Taiwan, R.O.C. He is also with the National Nano Device Laboratories, Hsinchu 300, Taiwan, R.O.C. (e-mail: scwang@mail.ndl.org.tw).

P. Su is with the Department of Electronics Engineering, National Chiao Tung University, Hsinchu 300, Taiwan, R.O.C. (e-mail: pinsu@ mail.nctu.edu.tw).

K.-M. Chen and G.-W. Huang are with the National Nano Device Laboratories, Hsinchu 300, Taiwan, R.O.C.

C.-T. Lin and V. Liang are with the United Microelectronics Corporation, Hsinchu 300, Taiwan, R.O.C.

Color versions of one or more of the figures in this letter are available online at http://ieeexplore.ieee.org.

Digital Object Identifier 10.1109/LMWC.2008.2001013
RF MOSFET. The popular van der Ziel's model is also used to check its applicability at different temperatures. Along with the extracted small-signal and van der Ziel's model parameters, their temperature dependences can be well described. Finally, their bias dependence results as a function of temperature are also presented.

\section{DEVICES AND MEASUREMENTS}

The RF MOSFETs used in this study were fabricated using UMC $0.13 \mu \mathrm{m}$ bulk technology. The transistor's gate length, finger length, finger number and group number are $0.36 \mu \mathrm{m}$, $3.6 \mu \mathrm{m}, 16$ and 2 , respectively.

The noise parameters of the device under different temperatures were measured using ATN NP5B noise parameter measurement system. The pads and series parasitics were de-embedded to obtain the intrinsic noise parameters. Finally, the intrinsic noise current sources were extracted by following the approach presented in [5].

\section{RESULTS AND DISCUSSIONS}

The van der Ziel's model widely adopted to characterize $S_{i g}, S_{i d}$, and $S_{i g d^{*}}$ can be expressed as follows [6]:

$$
\begin{aligned}
S_{i g} & =\frac{\overline{i_{g} i_{g}^{*}}}{\Delta f}=\delta 4 k T \frac{\omega^{2} C_{0}^{2}}{g_{d 0}} \\
S_{i d} & =\frac{\overline{i_{d} i_{d}^{*}}}{\Delta f}=\gamma 4 k T g_{d 0} \\
S_{i g d^{*}} & =\frac{\overline{i_{g} i_{d}^{*}}}{\Delta f}=j \varepsilon 4 k T \omega C_{0}
\end{aligned}
$$

where $C_{0}$ is the gate capacitance $\left(=3 C_{\mathrm{gs}} / 2\right), g_{d 0}$ is the channel conductance at zero drain bias, $k$ is Boltzmann constant, and $T$ is the ambient temperature in Kelvin. A fairly good data-model comparison of $S_{i g}, S_{i d}$ and $S_{i g d^{*}}$ for the device under study biased at $V_{\mathrm{GS}}=V_{\mathrm{DS}}=1.2 \mathrm{~V}$ can be obtained and are shown in Figs. $1-3$, respectively.

In these figures, one can find that $S_{i g}$ and $S_{i g d^{*}}$ would become larger for higher ambient temperature. For $S_{i d}$, however, it tends to decrease with increasing temperature. To explain these different trends, Table I lists the extracted $C_{0}, g_{d 0}$ and their normalizations with respect to their cases at $-40^{\circ}$. Besides, the extracted model parameters $\delta, \gamma$ and $\varepsilon$ for different temperatures are also shown in Fig. 4. It is also worth noting that the van der Ziel's model was originally derived for long channel devices, and the model parameters should be $\delta_{\text {sat }}=16 / 135, \gamma_{\text {sat }}=2 / 3$, and $\varepsilon_{\text {sat }}=1 / 9$ in the saturation region. It is no surprise that for 


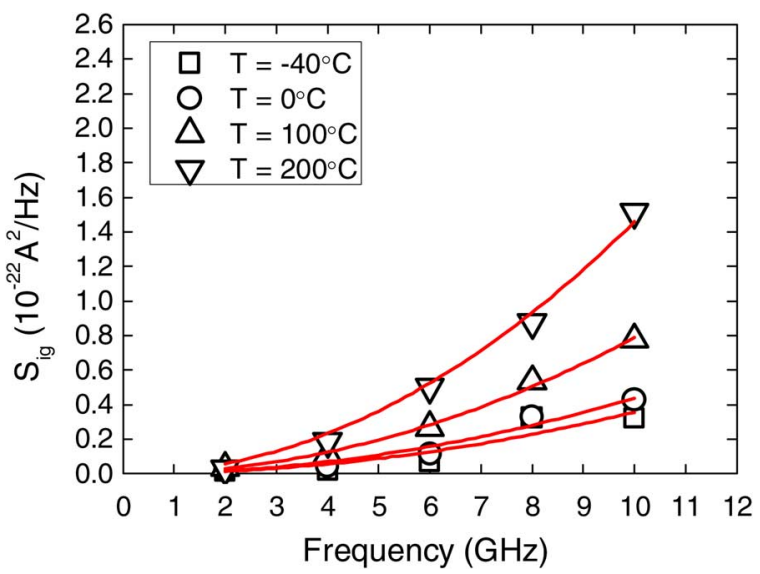

Fig. 1. Induced gate noise $\left(S_{i g}\right)$ versus frequency under different temperatures. $\left(V_{\mathrm{GS}}=V_{\mathrm{DS}}=1.2 \mathrm{~V}\right)$.

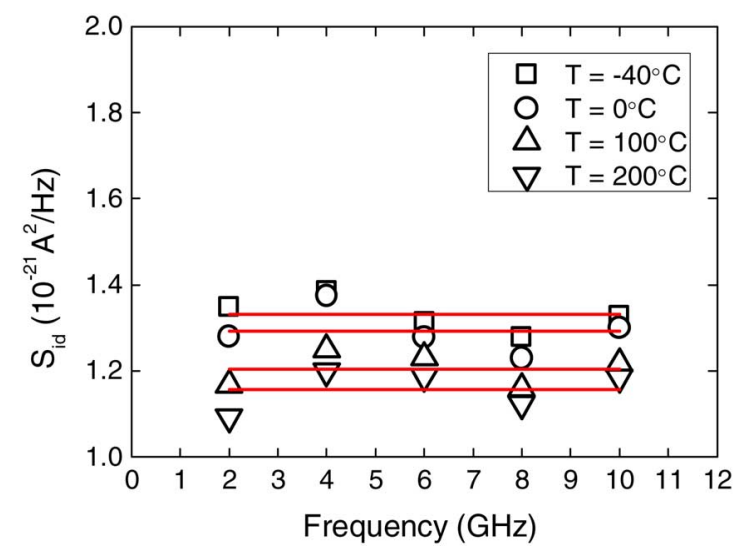

Fig. 2. Channel noise $\left(S_{i d}\right)$ versus frequency under different temperatures. $\left(V_{\mathrm{GS}}=V_{\mathrm{DS}}=1.2 \mathrm{~V}\right)$.

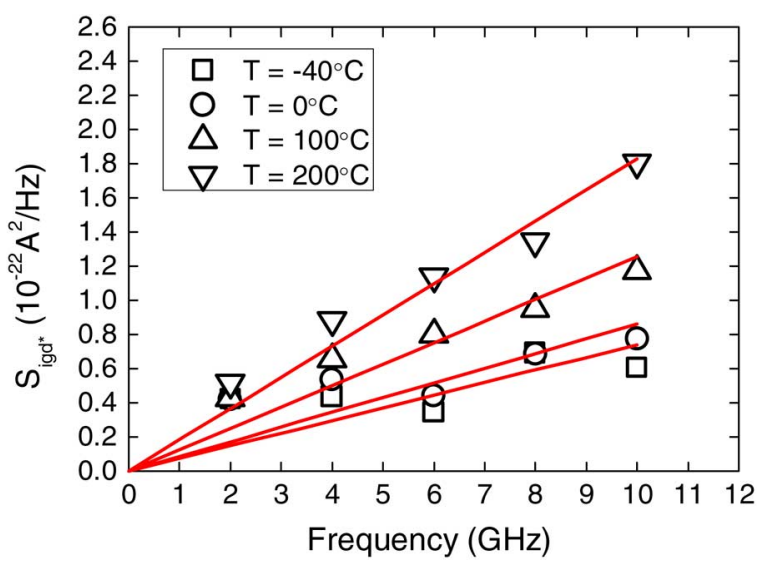

Fig. 3. Correlation noise $\left(S_{i g d *}\right)$ versus frequency under different temperatures. $\left(V_{\mathrm{GS}}=V_{\mathrm{DS}}=1.2 \mathrm{~V}\right)$.

TABLE I

EXTRACTED $g_{d 0}, C_{0}$ AND THEIR NORMALIZATIONS WiTH RESPECT TO CASES AT $-40^{\circ}$

\begin{tabular}{|c|c|c|c|c|c|c|}
\hline & $T(\mathrm{~K})$ & $\|T(\mathrm{~K})\|$ & $\begin{array}{l}g_{d 0} \\
(\mathrm{mS})\end{array}$ & $\left\|g_{d 0}\right\|$ & $\begin{array}{l}C_{0} \\
(\mathrm{fF})\end{array}$ & $\left\|C_{0}\right\|$ \\
\hline$T=-40^{\circ} \mathrm{C}$ & 233 & $\times 1$ & 112.4 & $\times 1$ & 520 & $\times 1$ \\
\hline$T=0{ }^{\circ} \mathrm{C}$ & 273 & $\times 1.17$ & 96 & $\times 0.85$ & 517 & $\times 0.99$ \\
\hline$T=100{ }^{\circ} \mathrm{C}$ & 373 & $\times 1.60$ & 66 & $\times 0.59$ & 508 & $\times 0.98$ \\
\hline$T=200^{\circ} \mathrm{C}$ & 473 & $\times 2.03$ & 51 & $\times 0.45$ & 506 & $\times 0.97$ \\
\hline
\end{tabular}

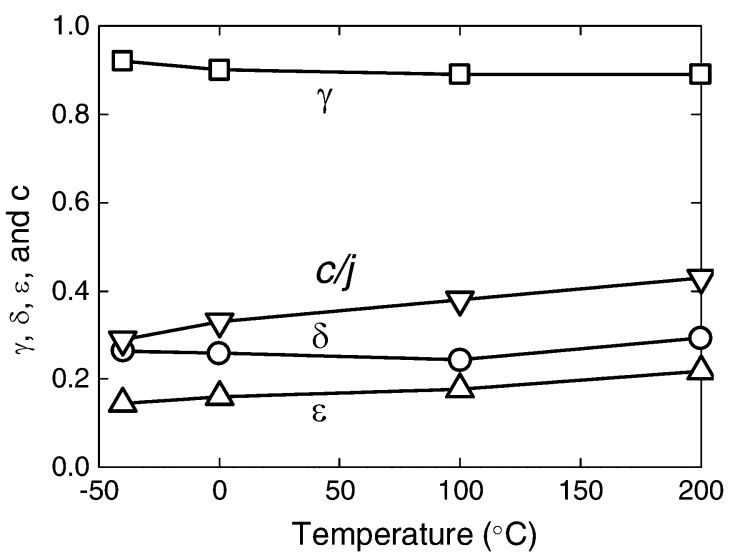

Fig. 4. Model parameters $\delta, \gamma, \varepsilon$ and correlation coefficient versus temperature.

the short channel device as in our study, the parameters could deviate from these theoretical values [5], [7], [8].

The small variations for $\delta, \gamma$ and $\varepsilon$ shown in Fig. 4 reveal that they are less temperature-dependent, and may not be the main contribution to the temperature dependence of these three PSDs. In addition, $C_{0}$ in Table $\mathrm{I}$ is shown to be insensitive to temperature. Therefore, for a given operating frequency, the following approximations can be achieved:

$$
\begin{aligned}
S_{i g} & \propto \frac{T}{g_{d 0}} \\
S_{i d} & \propto T g_{d 0} \\
S_{i g d^{*}} & \propto T .
\end{aligned}
$$

Equation (6) directly captures the positive temperature coefficient observed for $S_{i g d^{*}}$.

On the other hand, as temperature increases, the channel mobility would decline [2], causing $g_{d 0}$ to decrease with increasing temperature as shown in Table I. This explains the positive temperature coefficient for $S_{i g}$ (4). Moreover, since the decrease of $g_{d 0}$ overwhelms the increase of ambient temperature in Kelvin (Table I), $S_{i d}$ would have negative temperature coefficient (5). Besides, the correlation coefficient between noise currents $i_{g}$ and $i_{d}$ (denoted as) can be expressed as

$$
c \equiv \frac{\overline{i_{g} i_{d}^{*}}}{\sqrt{\overline{i_{g} i_{g}^{*}} \cdot \overline{i_{d} i_{d}^{*}}}}=j \frac{\varepsilon}{\sqrt{\delta \gamma}} .
$$

Since $\delta, \gamma$, and $\varepsilon$ are shown to be less temperature-dependent, the temperature dependence of shown in Fig. 4 is also weak.

Finally, the extracted values for the model parameters and the correlation coefficient for various gate and drain biases are shown in Fig. 5(a)-(d). It suggests that in the wide temperature range between $-40^{\circ}$ and $200^{\circ}, \delta, \varepsilon$, and $c / j$ have a greater temperature dependence at higher $V_{\mathrm{GS}}$, while $\gamma$ has a greater temperature dependence only for a lower $V_{\mathrm{GS}}$.

\section{CONCLUSION}

In this letter, we have investigated the temperature dependence of $S_{i g}, S_{i d}$ and $S_{i g d^{*}}$ for the RF MOSFET. $S_{i g}$ and $S_{i g d^{*}}$ are found to have positive correlation with ambient temperature, while $S_{i d}$ has negative one due to much lower channel conductance at higher temperature. Our experimental results show that 


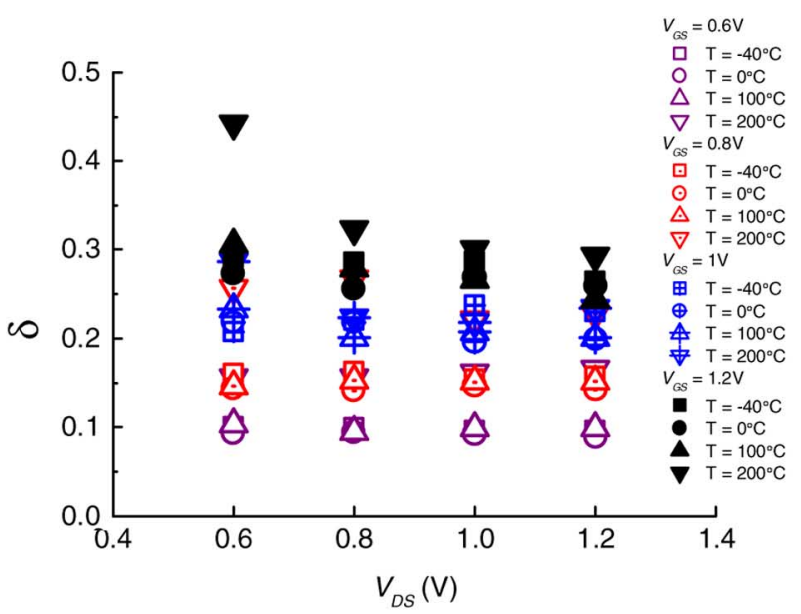

(a)



(c)



(b)

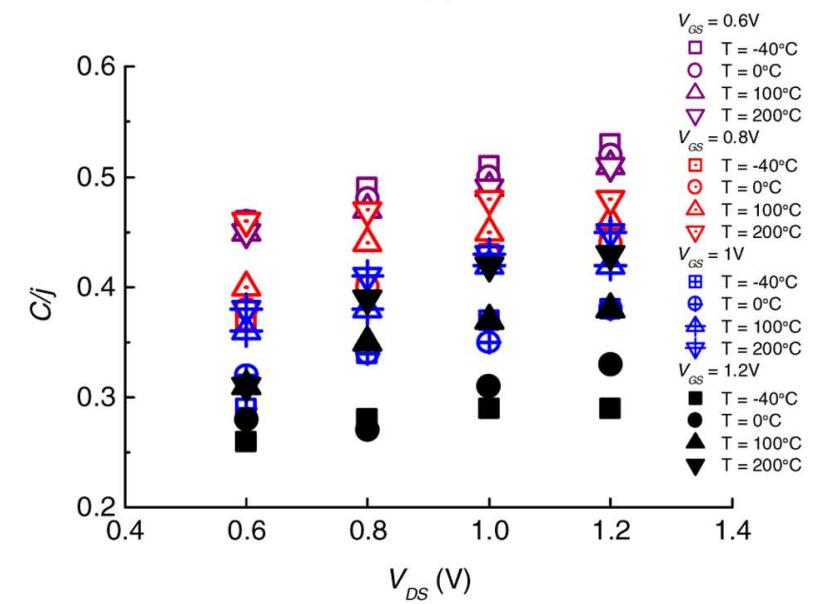

(d)

Fig. 5. Noise model parameters (a) $\delta$, (b) $\gamma$, (c) $\varepsilon$, and (d) $c / j$ versus drain bias for different temperature and gate bias conditions.

an accurate temperature model of the channel conductance is crucial to the RF noise temperature modeling.

\section{REFERENCES}

[1] A. J. Scholten, L. F. Tiemeijer, R. van Langevelde, R. J. Havens, A. T. A. Zegers-van Duijnhoven, and V. C. Venezia, "Noise modeling for RF CMOS circuit simulation," IEEE Trans. Electron Devices, vol. 50, no. 3, pp. 618-632, Mar. 2003.

[2] S. M. Nam, B. J. Lee, S. H. Hong, C. G. Yu, J. T. Park, and H. $\mathrm{K}$. Yu, "Experimental investigation of temperature dependent RF performances of RF-CMOS devices," in Proc. 6th Int. Conf. VLSI CAD (ICVC'99), 1999, pp. 174-177.

[3] Y. S. Lin, "Temperature dependence of the power gain and scattering parameters $s 11$ and $s 22$ of an RF nMOSFET with advanced RF-CMOS technology," Microw. Opt. Technol. Lett., vol. 44, no. 2, pp. 180-185, Jan. 20, 2005.
[4] A. Pascht, M. Grozing, D. Wiegner, and M. Berroth, "Small-signal and temperature noise model for MOSFETs," IEEE Trans. Microw. Theory Tech., vol. 50, no. 8, pp. 1927-1934, Aug. 2002.

[5] C.-H. Chen, M. J. Deen, Y. Cheng, and M. Matloubian, "Extraction of the induced gate noise, channel noise, and their correlation in submicron MOSFETs from RF noise measurements," IEEE Trans. Electron Devices, vol. 48, no. 12, pp. 2884-2892, Dec. 2001.

[6] A. van der Ziel, Noise in Solid State Devices and Circuits. New York: Wiley, 1986.

[7] K. Han, H. Shin, and K. Lee, "Analytical drain thermal noise current model valid for deep submicron MOSFET's," IEEE Trans. Electron Devices, vol. 51, no. 2, pp. 261-269, Feb. 2004.

[8] K. Han, J. Gil, S.-S. Song, J. Han, H. Shin, C.-K. Kim, and K. Lee, "Complete high frequency thermal noise modeling of short-channel MOSFETs and design of $5.2 \mathrm{GHz}$ low noise amplifier," IEEE J. SolidState Circuits, vol. 40, no. 3, pp. 726-269, Mar. 2005. 